\title{
Congenital Aniridia Associated with Congenital Aphakia and Glaucoma: A Case Report
}

\author{
Shray Dogra ${ }^{1}$, Pawan Prasher $^{2}$
}

\begin{abstract}
Introduction: Congenital aniridia is a rare disease associated with absent irides and various other ocular and systemic conditions. We report an extremely rare incidence of congenital aniridia associated with congenital aphakia and glaucoma in a young man.

Case description: A 28-year-old man presented with symptoms of ocular discomfort and low vision in his left eye. Visual acuity was finger counting at a half-meter, and examination showed the presence of aniridia, aphakia, nystagmus, and aniridic keratopathy, along with partial glaucomatous optic atrophy, poor foveal reflex, and a few white vitreous opacities in the fundus. The management was done conservatively with aphakic glasses, lubricants, and antiglaucoma drops.

Conclusion: The management of congenital aphakia associated with other ocular anomalies should be aimed at the preservation of residual vision and prevention of sight-threatening complications.

Keywords: Aniridia, Aphakia, Glaucoma, Keratopathy, Nystagmus.

AMEl's Current Trends in Diagnosis \& Treatment (2021): 10.5005/jp-journals-10055-0120
\end{abstract}

\section{INTRODUCTION}

Congenital aniridia is a rare eye disease characterized by the absence of iris in both eyes. The most common inheritance pattern seen is autosomal dominant followed by sporadic inheritance. Autosomal recessive inheritance is seen in a very small fraction of cases. Mutation of the paired box 6 (PAX6) gene has been most prominently demonstrated in this condition, and congenital aniridia is often associated with various other ocular and systemic anomalies, including keratopathy, glaucoma, hearing loss, and mental retardation. ${ }^{1}$

However, the association of congenital aniridia with congenital aphakia is very rare and only three cases have been reported to date. ${ }^{2-4}$ The chance of association between congenital aniridia and congenital aphakia has been estimated to be 1 in 490 million live births. Reported herein, is another patient of this very rare entity.

\section{Case Description}

We report a case of a 28-year-old man who presented to the ophthalmology clinic with symptoms of ocular surface discomfort and low vision in the left eye for the last one year. There was no associated redness, watering, or discharge present in the left eye. The right eye had been lost to a corneal ulcer in childhood and had become phthisic. Medical and treatment histories of the patient were not significant in the context of his symptoms, and there was no history of the presence of a similar disease in any of the family members.

Visual acuity in the left eye was finger counting at a half-meter on the Snellen acuity testing, and horizontal jerk nystagmus was observed on examination. Intraocular pressure (IOP) measured by applanation tonometry was $25 \mathrm{~mm}$ of $\mathrm{Hg}$ in the left eye. Slitlamp examination of the anterior segment was remarkable for the presence of aniridia, aphakia, and aniridic keratopathy in the left eye (Fig. 1A). Retroillumination demonstrated the presence of vitreous membranes posteriorly (Fig. 1B). Fundus examination showed partial glaucomatous optic atrophy with a cup-to-disk ratio of 0.8 , along with a poor foveal reflex, and a few white vitreous
1,2Department of Ophthalmology, Sri Guru Ram Das Institute of Medical Sciences and Research, Amritsar, Punjab, India

Corresponding Author: Pawan Prasher, Department of Ophthalmology, Sri Guru Ram Das Institute of Medical Sciences and Research, Amritsar, Punjab, India, Phone: +91 9646104858, e-mail: pawanprasher@yahoo.com

How to cite this article: Dogra S, Prasher P. Congenital Aniridia Associated with Congenital Aphakia and Glaucoma: A Case Report. AMEI's Curr Trends Diagn Treat 2021;5(1):53-54.

Source of support: Nil

Conflict of interest: None

opacities (Fig. 1C). Systemic examination was unremarkable. B-scan ultrasound examination showed an axial length of $25 \mathrm{~mm}$ of the eyeball along with the absence of iris and lens. Hyperechoic membranes were seen floating in the vitreous suggestive of vitreous degeneration (Fig. 1D). A computed tomography scan showed no evidence of a Wilms' tumor of the kidney. In view of the monocular status and guarded visual prognosis, the patient was treated conservatively with aphakic correction by tinted glasses for his low vision, along with topical lubricants for symptoms of ocular discomfort. Topical antiglaucoma drops, including a combination of timolol with brimonidine and dorzolamide, were added to lower the IOP. The patient was advised for regular follow-up and reported no worsening of vision or progression of glaucoma for 1 year.

\section{Discussion}

Congenital aniridia is a rare ocular disease usually caused by mutations in the PAX6 gene located on chromosome 11p13. Twothirds of aniridia cases are inherited in an autosomal, dominant fashion with variable expressivity, and the other cases are sporadic. It could not be established whether aphakia in the current case was primary or secondary. Previous authors have speculated that 

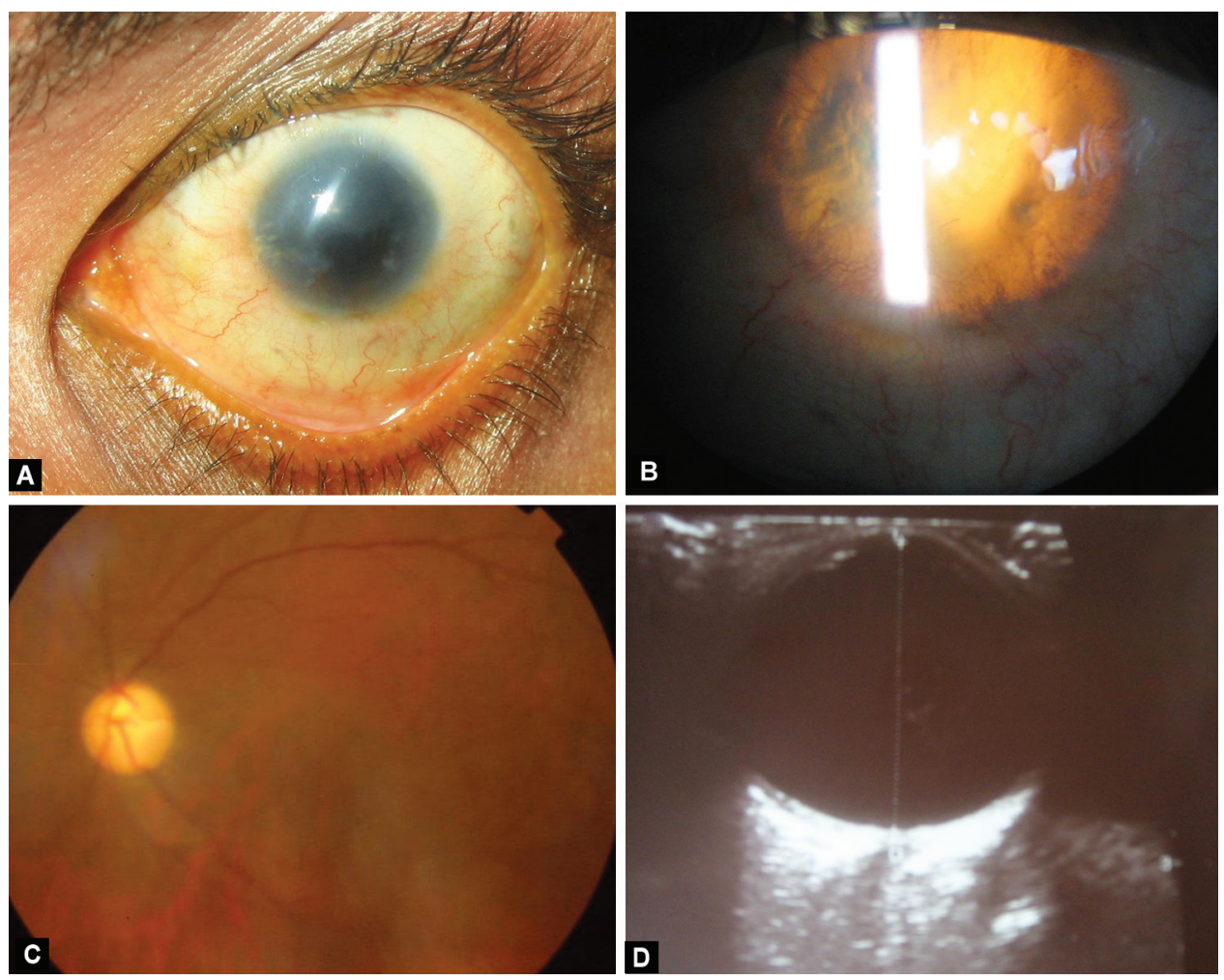

Figs $1 A$ to $D$ : (A) Left eye showing the absence of iris and the presence of aniridic keratopathy in the form of irregular epithelium near the limbus; (B) Retroillumination shows aniridia with few membranes floating in the vitreous (left side of the slit beam); (C) Fundus examination showed an increased cup-to-disk ratio of 0.8 and inferior notching; (D) B-scan showed the absence of iris and lens with few hyperechoic membranes floating in vitreous suggestive of vitreous degeneration

it could be a result of spontaneous absorption of the crystalline lens at an early age. ${ }^{1,5,6}$ The surgical management of this type of glaucoma is challenging. Filtering or drainage implant surgery with vitrectomy, goniosurgery, or cyclodestructive surgery can be effective. However, the efficacy of any of these treatments has not been established due to the extremely rare occurrence of this entity. Our patient responded well to conservative treatment with topical lubricants and tinted glasses, leading to improvement in the symptoms of ocular discomfort and a best-corrected visual acuity of 6/60. IOP was controlled on a combination of timolol with brimonidine and dorzolamide. Since the results of surgery (glaucoma or secondary intraocular implantation) in aniridia can be unpredictable and it is the only eye of the patient, it was decided to continue with conservative medical treatment along with close monitoring of glaucomatous damage for the time being.

\section{CONCLUSION}

As congenital aniridia may be associated with various other ocular anomalies of varying severities, ophthalmological management should be aimed at the preservation of residual vision and the prevention of sight-threatening complications.

\section{References}

1. Tripathy K, Salini B. Aniridia. In: StatPearls. Treasure Island, FL: StatPearls Publishing, 2021. Available at: http://www.ncbi.nlm.nih. gov/books/NBK538133/

2. Moreker M, Parikh R, Parikh SR, et al. Aniridia associated with congenital aphakia and secondary glaucoma. Indian J Ophthalmol 2009;57(4):313. DOI: 10.4103/0301-4738.53061.

3. Terasaki $H$, Yamashita $T$, Tanaka $M$, et al. Aniridia associated with aphakia and secondary glaucoma. Jpn J Ophthalmol 2010;54(5): 504-505. DOI: 10.1007/s10384-010-0852-7.

4. Yoshikawa $\mathrm{K}$, Asakage $\mathrm{H}$, Inoue Y. Membranous cataract in association with aniridia. Jpn J Ophthalmol 1993;37(3):325-329. Available at: https://pubmed.ncbi.nlm.nih.gov/8295372/

5. Nelson LB, Spaeth GL, Nowinski TS, et al. Aniridia. A review. Surv Ophthalmol 1984;28(6):621-642. DOI: 10.1016/00396257(84)90184-x.

6. Hingorani M, Hanson I, van Heyningen V. Aniridia. Eur J Hum Genet 2012;20(10):1011-1017. DOI: 10.1038/ejhg.2012.100. 